\title{
Formation and evolution of carbon-enhanced metal-poor stars
}

\author{
T. Suda ${ }^{* a}$, Y. Komiya ${ }^{a}$, S. Yamada $^{b}$, W. Aoki ${ }^{c}$, and M. Y. Fujimoto ${ }^{d}$ \\ Research Center for the Early Universe, The University of Tokyo \\ Hongo 7-3-1, Bunkyo-ku, Tokyo 113-0033, Japan \\ $b$ \\ Department of Cosmosciences, Hokkaido University \\ Kita 10 Nishi 8, Kita-ku, Sapporo 060-0810, Japan
}

TMT Project Office, National Astronomical Observatory of Japan

Osawa 2-21-1, Mitaka, Tokyo 181-8588, Japan

$d$

Faculty of Engineering Hokkai-gakuen University

Asahimachi 4-1-40, Toyohira-ku, Sapporo 062-8605, Japan

E-mail: sudadresceu.s.u-tokyo.ac.70

Carbon-enhanced metal-poor (CEMP) stars play a key role in characterising the star formation history of the Galactic halo. In particular, most stars show carbon enhancement among the lowest metallicity range. In this paper, we explore the origin of the three most iron-poor stars (called hyper metal-poor or HMP stars) with $[\mathrm{Fe} / \mathrm{H}]<-5$ (which are all CEMP stars) in terms of binary hypothesis. We examined the metallicity distribution function of extremely metal-poor stars $([\mathrm{Fe} / \mathrm{H}] \lesssim-2.5)$ using the merger tree models with chemical evolution. We also tried to reproduce the abundance patterns of these stars using the nucleosynthesis models of a hydrogen ingestion into the helium-flash convective zones of metal-free AGB stars. Both experiments suggest that the most iron-poor stars are likely to be the first generation stars born out of primordial gas with their surface polluted by the mass loss wind from AGB stars and the ejecta of supernovae in the early universe.

XIII Nuclei in the Cosmos,

7-11 July, 2014

Debrecen, Hungary

\footnotetext{
* Speaker.
} 


\section{Introduction}

Extremely metal-poor stars should bear the important imprints of the early universe. Observations reveal that the frequency of carbon-enhanced metal- poor (CEMP) stars is increasing with decreasing metallicity, [Fe/H] [U, [2], thanks to the follow-up observations of metal-poor stars such as the HK survey [3] and the Hamburg-ESO survery [田]. In particular, all of the so-called hyper metal-poor (HMP) stars with $[\mathrm{Fe} / \mathrm{H}]<-5$ are CEMP stars [ [0, $6, \mathbf{6}, \mathbf{\square}]$. The origin of these stars is still a matter of debate. Proposed scenarios are faint supernovae [ [8], fast-rotating massive stars [Q], and binary mass transfer from AGB stars [ㅁ]]. We have proposed that carbon and many other elements in CEMP (both CEMP-s and CEMP-no subclasses, which is devided by the abundance of

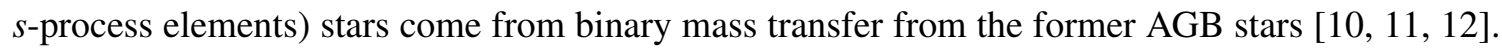
This scenario inevitably requires the high- mass stellar initial mass function (IMF) for population

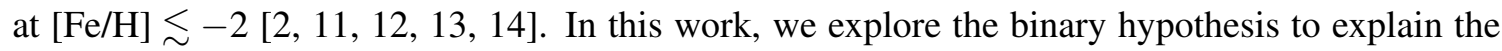
abundance pattern of the carbon-enhanced HMP stars including the most iron-deficient stars SMSS J031300 reported recently [ []].

\section{Models and Assumptions}

To predict the metallicity distribution function (MDF) of the Galactic halo stars, we construct a realisation of the Milky Way halo with $2 \times 10^{12} M_{\odot}$ based on the hierarchical clustering scenario [106, [7]. Star formation rate is set to be proportional to the gas mass of mini halos while the star formation efficiency is set constant, where the minimum mass of mini halo is determined by the virialized temperature of $T_{\mathrm{vir}}=10^{3} \mathrm{~K}$. Adopted stellar IMF is based on our previous work, i.e., the log-normal function with a median mass of $10 M_{\odot}$ and a variance of 0.4 [ㅍ], eq.(36)], which gives a good fit to the observed fraction of CEMP stars. All the individual stars are registered in the simulations together with the information about iron abundances that is a consequence of chemical evolution in individual halos. We consider the effects of (1) the surface pollution of stars with metals, caused by the accretion of metals by first supernova ejecta in host halos, and (2) the pre-enrichment of metals in the host halo polluted by external supernova ejecta. Predicted MDF provides the origin of the most iron-poor stars whether they are polluted Pop. III or the second generation stars formed out of gas polluted by the ejecta of the first supernovae.

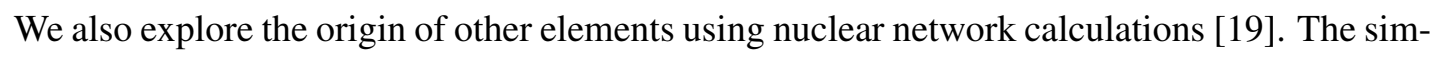
ulation is based on analytic formalae to describe the evolution of helium shell flashes [22]. Using this recipe, we can trace the abundances in the helium flash convective zones by considering the nuclear reactions at its bottom. In our nuclear network, we include all the reactions for 318 element species from neutron to sulphur and neutron-capture reactions up to polonium. We followed the nucleosynthesis of a proton ingestion into the helium-flash convective zones during the TPAGB phase that happens for stars with $M \lesssim 3 M_{\odot}$ and [Fe/H] $\lesssim-2.5$ [2], 23], 24] as well as the $s$-process by ${ }^{22} \mathrm{Ne}$ in massive AGB stars. The former mixing and the subsequent dredge-ups (Helium-Flash Driven Deep Mixing, He-FDDM) are responsible for the production of CNO, light, and $s$-process elements [एव, [2]]. The evolution of density and temperature at the bottom of helium-flash convective zones are approximated by one-zone models [ए]], which allows us to survey large parameter range for nucleosynthesis in Pop. III and low metallicity AGB stars. 

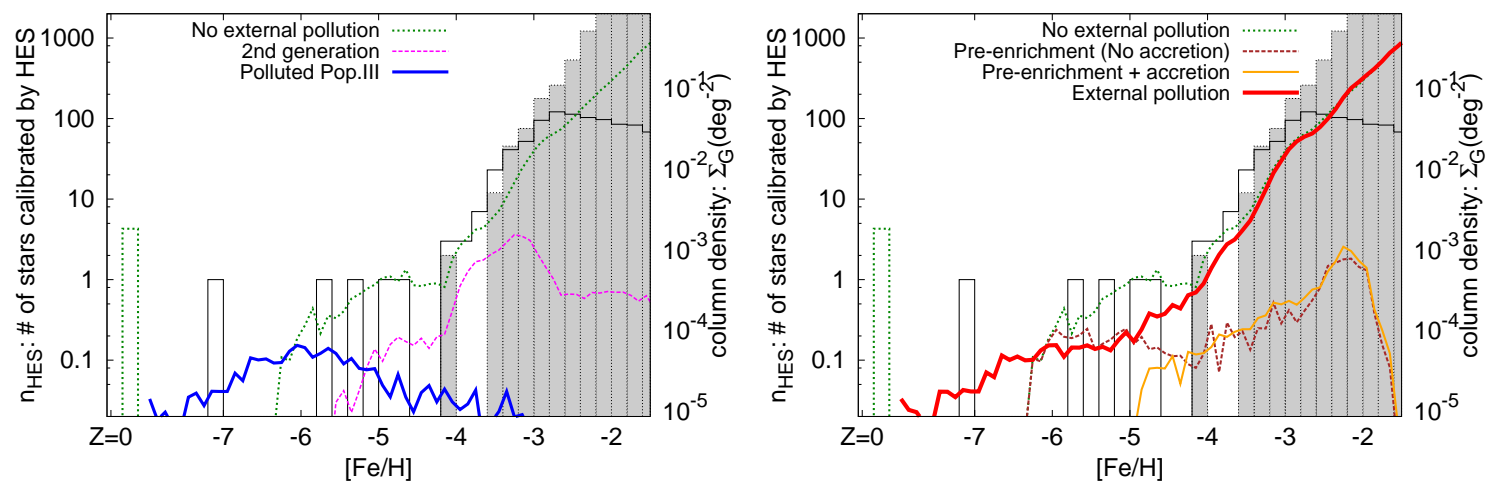

Figure 1: Comparisons of observed (histogram) and predicted (lines) distribution of metal-poor stars in the Galactic halo. Number of stars predicted by models is calibrated by the survey area of the Hamburg-ESO survey (HES). We considered the contribution of metal-pollution by the accretion of interstellar medium and the pre-enrichment of the first host clouds (see text).

\section{Effect of Accretion and Pollution}

The metallicity distribution of the Galactic halo stars can be the result of chemical evolution in individual host mini haloes that build up the current system. For simplicity, the supernova ejecta from massive stars are well mixed in the host halo, which determines the metallicity of the next generation stars. Considering the effect of surface accretion and pre-enrichment according to the description in the previous section, our merger tree models for Galactic chemical evolution [ㅍ] imply that the iron-group elements of HMP stars come from interstellar pollution, not from either second generation stars or pre-enrichment by external supernovae [18]]. As stated above, if we ignore the effect of interstellar accretion, intrinsic population includes $\sim 10$ Pop.III stars (metal-free and first generation stars) in the current survey area, which is larger than expected from observations [ए]]. This is simply because all the star formation begins with Pop. III stars and mass spectrum contains low-mass survivors even with the assumption of high-mass dominated IMF. This is shown by dotted line in the left panel of Figure $\mathrm{W}$. The thick solid line represents the distribution of Pop. III stars whose surfaces are polluted by interstellar accretion.

We also consider the effect of pollution in the inter-Galactic medium [ए8]]. We assume that metal-free gas can be polluted by supernova ejecta before the onset of the formation of host haloes. Such gas clouds produce pre-enriched first generation stars whose distribution is shown by the dashed line in the right panel of Fig. $\mathrm{W}$. The solid line in the figure shows the distribution of preenriched first generation stars with the effect of interstellar accretion taken into consideration. The total distribution of first generation stars influenced by external pollution (pre-enrichment and/or surface accretion) is shown by the thick solid line in the right panel.

Compared with the distribution of polluted Pop. III stars (first generation stars with their surface polluted by metals, while the inside of stars is metal-free), second generation stars (dashed line in the left panel), and pre-enriched first generation stars, it is likely that the stars with $[\mathrm{Fe} / \mathrm{H}]<-5$ are polluted Pop. III rather than later generations of stars. For stars with $-5 \lesssim[\mathrm{Fe} / \mathrm{H}] \lesssim-4$, they are originated from second generation stars formed out of gas polluted by Pop.III supernovae in 

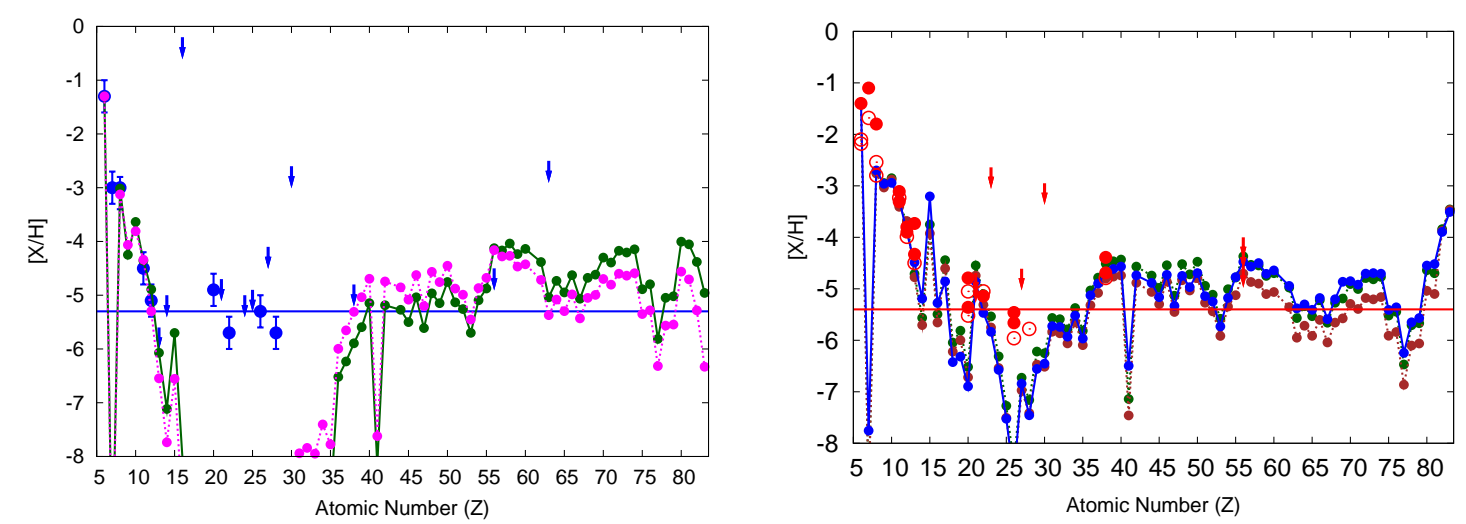

Figure 2: Comparsions of observed (symbols and arrows) and predicted abundance patterns (circles connected by lines) for HE 0107-5240 (left) and HE 1327-2326 (right). Filled symbols are abundances analysed by 1D LTE models, while open symbols are those with 3D corrections. Arrows denote upper limits. Solid line shows the metallicity of each star. For HE 0107-5240, we find two models to match the abundance patterns. These models adopt the same parameter set for mixing, while the adopted stellar models are different. For HE 1327-2326, we show three lines with different mixing timescales.

the same host cloud, or from first generation stars formed out of gas pre-enriched by external supernovae.

\section{Nucleosynthsis in AGB Stars}

Abundance patterns of known HMP stars are compared with the results of the nucleosynthesis in metal-free AGB stars using s-process nucleosynthesis code [ए耳]. We assumed a hydrogen ingestion into the helium flash convective zones during the thermal pulse phase of intermediate-mass metal-free stars. Initial models are taken from the results of our previous work [20, 201].

There are several parameters to describe the mixing. In this study, we assumed that the mixing event depends on the amount of mixed hydrogen, the duration of a mixing event, the properties of the helium-flash convective zones (mass and radius of helium core at the onset of mixing), the supply of metals from the envelope to the helium-flash convective zones, and the efficiency of dredge-up. Note that stellar models are not characterised by stellar mass (or core mass), but by the maximum temperature at the bottom of the hydrogen-flash convective zones triggered by the hydrogen mixing. Therefore, it is difficult to specify progenitor masses for our models, although, in general, the higher the maximum temperature, the larger the initial mass. The final abundances after dredge-ups are scaled to match the observed carbon abundances.

Figures $\square$ and 3 show the comparisons of models with HE 0107-5240, HE 1327-2326, and SMSS J0313. The abundance patterns of these HMP stars can be satisfactorily reproduced by various channels of AGB evolution. For HE 0107-5240, the abundance patterns can be well reproduced by the He-FDDM with small amount of hydrogen mixing and possible contamination of metals during the second dredge-up for $M>2.5 M_{\odot}$ [प] ]. The abundances of HE 1327-2326 can be reproduced by the He-FDDM as well, while the amount of mixing is larger than the case for HE 0107-5240. The most iron-poor star, SMSS J0313, may have nucleosynthetic signature of $s$-process triggered by ${ }^{22} \mathrm{Ne}$. The $\mathrm{CNO}$ abundances can be explained by the third dredge-up. 


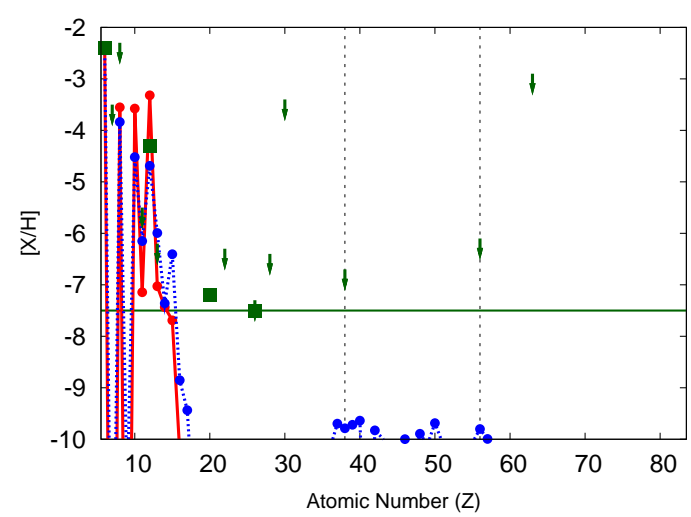

Figure 3: Same as Fig. 2, but for SMSS J0313. Two different models represent different stellar models with the same amount of mixing and the duration of a mixing event.

\section{Conclusions and future work}

Origins of the most iron-poor stars have been explored based on the scenario of binary mass transfer, nucleosynthesis, and the hierarchical Galaxy formation. We propose that the abundance patterns of three most iron-poor (hyper metal-poor, HMP) stars are the consequence of nucleosynthesis in AGB stars and pollution by interstellar medium, and hence these stars are polluted Pop. III stars.

However, there are open questions to be answered for our comprehensive understandings of the nature of CEMP stars including HMP stars that we have discussed: Why do CEMP-no stars (perhaps including HMP stars) show no evidence of binarity? Why do CEMP-s stars (definite contribution of binary mass transfer events) disappear at $[\mathrm{Fe} / \mathrm{H}]<-3.5$ ? These questions will give us an insight into the star formation history of the Galaxy and the hierarchical clustering in the early universe.

\section{Acknowledgments}

This work is partially supported by the JSPS Grant-in-Aid for Scientific Research (23224004).

\section{References}

[1] D. Yong et al. 2013, The most metal-poor stars. III. the metallicity distribution function and carbon-enhanced metal-poor fraction, ApJ 762, 27

[2] Y.-S. Lee, T. Suda, T. C. Beers, and R. J. Stancliffe 2014, Carbon-enhanced Metal-poor Stars in SDSS/SEGUE. II. Comparison of CEMP-star Frequencies with Binary Population-synthesis Models, ApJ 788, 131

[3] T. C. Beers, G. W. Preston, and S. A. Shectman 1985, A search for stars of very low metal abundance. I, AJ 90, 2089

[4] N. Christlieb et al. 2001, The stellar content of the Hamburg/ESO survey I. Automated selection of DA white dwarfs, A\&A 366, 898 
[5] N. Christlieb et al. 2002, A stellar relic from the early Milky Way, Nature 419, 904

[6] A. Frebel et al. 2005, Nucleosynthetic signatures of the first stars, Nature 438, 871

[7] S. C. Keller et al. 2014, A single low-energy, iron-poor supernova as the source of metals in the star SMSS J031300.36-670839.3, Nature 506, 463

[8] H. Umeda, and K. Nomoto 2003, First-generation black-hole-forming supernovae and the metal abundance pattern of a very iron-poor star, Nature 422, 871

[9] G. Meynet, S. Ekström, and A. Maeder The early star generations: the dominant effect of rotation on the CNO yields, A\&A, 447, 623

[10] T. Suda, M. Aikawa, M. N. Machida, M. Y. Fujimoto, and I. Iben, Jr. 2004, Is HE 0107-5240 A Primordial Star? The Characteristics of Extremely Metal-Poor Carbon-Rich Stars, ApJ 611, 476

[11] Y. Komiya, T. Suda, H. Minaguchi, T. Shigeyama, W. Aoki, and M. Y. Fujimoto 2007, The Origin of Carbon Enhancement and the Initial Mass Function of Extremely Metal-poor Stars in the Galactic Halo, ApJ 658, 367

[12] T. Suda et al. 2013, Transition of the stellar initial mass function explored using binary population synthesis, MNRAS 432, L46

[13] T. Suda et al. 2011, The Stellar Abundances for Galactic Archaeology (SAGA) data base - II. Implications for mixing and nucleosynthesis in extremely metal-poor stars and chemical enrichment of the Galaxy, MNRAS 412, 843

[14] S. Yamada, T. Suda, Y. Komiya, W. Aoki, M. Y. Fujimoto 2013, The Stellar Abundances for Galactic Archaeology (SAGA) Database - III. Analysis of enrichment histories for elements and two modes of star formation during the early evolution of the Milky Way, MNRAS 436, 1362

[15] T. Suda et al. 2008, Stellar Abundances for the Galactic Archaeology (SAGA) Database Compilation of the Characteristics of Known Extremely Metal-Poor Stars, PASJ 60, 1159

[16] Y. Komiya, A. Habe, T. Suda, and M. Y. Fujimoto 2009, Hierarchical Formation of the Galactic Halo and the Origin of Hyper Metal-Poor Stars, ApJ 696, L29

[17] Y. Komiya, A. Habe, T. Suda, and M. Y. Fujimoto 2010, Formation History of Metal-poor Halo Stars with the Hierarchical Model and the Effect of Interstellar Matter Accretion on the Most Metal-poor Stars, ApJ 717, 542

[18] Y. Komiya, S. Yamada, T. Suda, and M. Y. Fujimoto 2014, The New Model of Chemical Evolution of r-process Elements Based on the Hierarchical Galaxy Formation. I. Ba and Eu, ApJ 783, 132

[19] T. Nishimura, M. Aikawa, T. Suda, and M. Y. Fujimoto 2009, Oxygen and Light-Element Synthesis by Neutron-Capture Reactions in Metal-Free and Extremely Metal-Poor AGB Stars, PASJ, 61, 909

[20] M. Y. Fujimoto, Y. Ikeda, and I. Iben Jr. 2000, The Origin of Extremely Metal-poor Carbon Stars and the Search for Population III, ApJ 529, L25

[21] T. Suda, and M. Y. Fujimoto 2010, Evolution of low- and intermediate-mass stars with $[\mathrm{Fe} / \mathrm{H}] \leq-2.5, \mathrm{MNRAS}, \mathbf{4 0 5}, 177$

[22] M.Y. Fujimoto, M. Aikawa, and K. Katö 1999, A Model of Deep Mixing in Globular Cluster Red Giants, ApJ, 519, 733

[23] S.W. Campbell, and J. C. Lattanzio 2008, Evolution and nucleosynthesis of extremely metal-poor and metal-free low- and intermediate-mass stars I. Stellar yield tables and the CEMPs, A\&A, 490, 769

[24] H. H. B. Lau, R. J. Stancliffe, and C. A. Tout 2009, The evolution of low-metallicity asymptotic giant branch stars and the formation of carbon-enhanced metal-poor stars, MNRAS, 396, 1046 